\title{
Investigating the Aeroacoustic Properties of Kevlar Fabrics
}

\author{
Máté Szőke, William J. Devenport† Nandita Harił W. Nathan Alexanderæ \\ Virginia Tech, Blacksburg, VA, 24061, USA \\ Stewart A. L. Glegg $₫$ \\ Florida Atlantic University, Boca Raton, FL, 33431, USA
}

Ang Li, Rahul Vallabh,*Abdel-Fattah M. Seyam, ${ }^{\dagger \dagger}$
North Carolina State University, Raleigh, NC, 27606, USA

\begin{abstract}
The aeroacoustic properties of porous fabrics are investigated experimentally in an effort to find a porous fabric as an ideal interface between wind tunnel flow and quiescent conditions. Currently, the commercially available Kevlar type $\mathbf{1 2 0}$ fabric is widely used for similar applications, such as side-walls in hybrid anechoic wind tunnels or as a cover of phased microphone arrays. A total number of 8 fabrics were investigated, namely, four glass fiber fabrics, two plain weave Kevlar fabrics, and two modified plain Kevlar fabrics with their pores clogged. Two, custom-made rigs were used to quantify the transmission loss and self-noise of all eight fabrics. It was found that the pores serve as a low-resistance gateway for sound waves to pass through, hence enabling a low transmission loss. The transmission loss was found to increase with decreasing open area ratio while other fabric properties had a minor impact on transmission loss. The self-noise of the fabrics has also been evaluated and it was found that the thread density (thread per inch) is a primary factor of determining the frequency range of self-noise with the open area ratio potentially playing a secondary role in the self-noise levels. For both metrics, the mass per unit area seemed to play a minor role in the aeroacoustic performances of the fabrics. Finally, surface pressure measurements revealed that the commercially available plain Kevlar (type 120) has no quantifiable effect on the hydrodynamic pressure field passing over the fabric, suggesting that Kevlar behaves as a no-slip wall from the flow's perspective when no pressure difference is present on the two sides of the fabric.
\end{abstract}

\section{Introduction}

Aeroacoustic investigations have long been seeking an interface that could separate the flow field from the quiescent conditions or anechoic chambers. In hybrid-anechoic wind tunnels (HAWT), tensioned ballistic Kevlar is often placed as an interface between the flow inside the test section and the surrounding anechoic chambers ${ }^{17}$ 3imilarly, Kevlar and wire meshes have been used for covering in-flow phased (or beamforming) microphone arrays to separate flow noise from the microphones. ${ }^{4} 8$ More recently, Kevlar has also been used as an interface between boundary layer flow and metamaterials. ${ }^{[]}$Overall, porous fabrics (such as Kevlar, or

\footnotetext{
*Senior Research Associate, Kevin T. Crofton Department of Aerospace and Ocean Engineering, email: m.szoke@vt.edu

${ }^{\dagger}$ Crofton Professor in Engineering, Kevin T. Crofton Department of Aerospace and Ocean Engineering, AIAA Associate Fellow

${ }^{\ddagger}$ Graduate Research Assistant, Kevin T. Crofton Department of Aerospace and Ocean Engineering

$\S$ Assistant Professor, Kevin T. Crofton Department of Aerospace and Ocean Engineering

『Professor, Department of Ocean and Mechanical Engineering, AIAA Associate Fellow

" Postdoctoral Research Scholar, Wilson College of Textiles

** Research Assistant, Wilson College of Textiles

${ }^{\dagger \dagger}$ Charles A. Cannon Professor of Textiles, Department Head, Textile and Apparel, Technology and Management, Wilson College of Textiles
} 
wire mesh) may be seen as an interface efficiently separating the acoustic field from the flow field, potentially opening a new dimension for aeroacoustic experimentation.

From the perspective of HAWT facilities, the benefits of Kevlar walls (or windows) are relatively well understood. Most often, the lightest available Kevlar weave is used (Kevlar 120) in HAWTs since it is acoustically transparent by having low transmission loss, on the order of a few decibels below $20 \mathrm{kHz}$. While it is near-transparent to sound, it is almost impervious to flow. The underlying reason for both of these properties is associated with its low open area ratio (OAR), which usually ranges between $2 \%$ and $6 \%$. When compared to a conventional open-jet aeroacoustic facility, Kevlar walls in HAWTs greatly reduce liftinduced aerodynamic interference and eliminate the need for a jet catcher 1010 Kevlar windows also allow for a much larger test section length to width ratid $^{3}$ greatly increasing the signal-to-noise ratio of acoustic measurements made with phased microphone arrays. The aerodynamic response of Kevlar to the flow stems from its membrane-like behavior,1010 in other words, Kevlar lacks bending stiffness hence it can efficiently follow the aerodynamic pressure field. The technology of HAWT thus has the potential to substantially enhance the capabilities of many aeroacoustic facilities, and indeed there has been a proliferation of facilities using Kevlar acoustic windows, such as the new Danish National Wind Tunnel $\frac{\sqrt[3]{3}}{\text { at }}$ CARDC in China, 11 NASA Langley ${ }^{[12}$ Florida State University, ${ }^{[3]}$ Beihang University, ${ }^{14}$ TU Delft, $\frac{15]}{\sqrt{15}}$ University of Bristol,2]16 and JAXA in Tokyo. 17

The wide scrutiny of this novel wind tunnel configuration has served primarily to confirm its desirable characteristics and to better understand its potential and limitations for further development. Brown et al $l^{[18}$ showed that the acoustic window deflection and in-plane strain field can be accurately measured optically. Sensing of the in-plane strain allows the tension distribution to be determined which, in turn, provides an estimate of the entire aerodynamic pressure field acting on the wind tunnel wall. Brown et al. demonstrated that this estimate was at least of sufficient accuracy to estimate the lift on a model placed in the test section.

In another development, using the Quiet Flow Facility at NASA Langley, Bahr et al ${ }^{12}$ showed that there are many benefits to using the Kevlar acoustic window configuration. One particular benefit is that Kevlar greatly reduces the thickness of the shear layers that form the boundaries of the flow. As such, it decreases the decoherence of sound waves as they pass through the shear layer, particularly at higher frequencies (above $10 \mathrm{kHz}$ ) and downstream observer locations. This is of critical importance to model scale testing of full aircraft configurations, such as it is routinely performed in national scale wind tunnel facilities for the development of large transport aircraft. A compensating problem was also uncovered, however. At similarly high frequencies (above $10 \mathrm{kHz}$ ) Kevlar can be a source of "scrubbing" noise. Studies of Kevlar fabric made backed with a solid surface or with a cavity $19 \mid 20$ indicated that this is not roughness noise, but noise generated by the pumping of air through the pores in the fabric, which is an efficient monopole mechanism. While Kevlar has a low transmission loss (a few decibels) at low frequencies (below $10 \mathrm{kHz}$ ), its acoustic losses may become significant at high frequencies (above $10 \mathrm{kHz}$ ) and can reach $10 \mathrm{~dB}\left[\mathbb{1}^{13}\right.$ From a HAWT or phased array application perspective, the presence of self-noise and high transmission loss at high frequencies (above $10 \mathrm{kHz}$ ) may compromise the aeroacoustic capabilities of HAWT facilities. Namely, these effects may restrict aeroacoustic measurements performed from behind the Kevlar windows in the anechoic chambers or could contaminate the measurements at high frequencies.

Only a limited amount of efforts have been made 1920 to understand the underlying physical problem associated with these drawbacks, which would be at the basis to overcome them. This paper, therefore, makes one of the first attempts to understand the underlying physical mechanisms of how Kevlar generates self-noise, while also quantifying self-noise and transmission loss (TL). The goal of the work presented here is to identify the properties of porous fabrics whose self-noise may be lower than, and transmission loss is comparable to that of Kevlar. Ultimately, the aim is to find an ideal fabric associated with low self-noise and low transmission loss even at higher frequencies (above $10 \mathrm{kHz}$ ), while maintaining the beneficial mechanical and aerodynamic properties of Kevlar. To reach these goals, this paper presents a detailed aeroacoustic characterization of different porous fabrics. The properties of the various fabrics are first introduced in Section A followed by the description of the experimental approach. Section III then provides a detailed discussion of the aeroacoustic results with findings summarized in Section IV]

\section{Experimental Approach}

Experiments were conducted at the wall jet wind tunnel (WJT) facility of Virginia Tech 21 to quantify the self-noise and transmission loss of various porous fabrics. Additional aeroacoustic testing has been 
performed to assess the effects of porous fabrics on the hydrodynamic pressure fluctuations within the turbulent boundary layer passing over them. For this reason, the surface pressure fluctuations have been measured upstream and downstream of the fabrics in the WJT. In addition, microscopic images of the fabrics have been taken to determine their open area ratio (OAR). The following paragraphs detail the experimental approaches for each of the measurements performed.

\section{A. Fabrics tested}

A total number of eight fabrics were tested whose properties are listed in Table 1 . Three different types of materials were tested: (a) glass fibers, (b) a commercially available Kevlar (K120-CST) and a custom-made Kevlar (K120-EAS, reduced OAR of $2.2 \%$ ), and (c) spray-on adhesive applied to K120-EAS resulting in a further reduced OAR and increased mass per unit area $(m)$ without changing the thread density of the fabric. Some properties listed in Table 1 were determined experimentally, while others were determined either analytically or obtained from manufacturer specifications. The OAR of the fabrics were determined both analytically ${ }^{22}$ using the weave type and yarn properties (denoted as calculated), and experimentally using microscopic images. In addition, the air permeability of the fabrics was also determined ${ }^{23}$ experimentally. Finally, the weight of the fabrics was taken from the manufacturer's specifications, except for K120-13 and K120-14 whose weight were determined using a high-precision scale (Intelligent Weighing Technology PM-100).

The properties of the tested fabrics listed in Table 1 determine important characteristics of the test matrix, namely, the various fabrics help us to quantify how the changes in open area ratio, thread density (TPI, thread per inch), and mass per unit area $(m)$ affect self-noise and transmission loss. Table 1 reveals that the glass fiber fabrics have a significantly higher OAR than Kevlar fabrics and about twice the thread density but a lower mass per unit area. These fabric samples can help to quantify the effects of OAR and TPI on transmission loss and self-noise. The commercially available Kevlar, K120-CST, may be considered as a baseline case of our investigations as it has been widely used in HAWT facilities. The K120-EAS fabric has been custom-made by EAS Fiberglass and it is currently used in the Stability Wind Tunnel of Virginia Tech. This fabric has a nominal OAR of $2.2 \%$, which is half the OAR of K120-CST, while it maintains all other properties of K120-CST. Again, a comparison between K120-EAS and K120-CST can shed light on the aeroacoustic effects of OAR. Finally, the K120-EAS fabric has been treated with Exceval RS-2117 type spray-on adhesive made by Kuraray America, Inc. to reduce its OAR and increase its mass per unit area $(m)$. Two levels of spray-on adhesive coating have been applied, a lower amount of spray has manually been applied to K120-13 while a higher amount of coating has been applied to K120-14. Note that the measured OAR values of these fabrics are inconsistent with the amount of coating applied which is due to the random distribution of unclogged pores. However, their air permeability is in agreement with the applied amount of spray-on adhesive. These observations also imply that the pore distribution of these fabrics (K120-13, K120-14) is random compared to the regular pore pattern that can be found on other fabrics tested in this study.

\begin{tabular}{c|c|c|c|c|c|c} 
Fabric ID & Fabric type & \multicolumn{2}{|c|}{ OAR (\%) } & $\begin{array}{c}\text { Air permeability } \\
(\text { CFM })\end{array}$ & $\begin{array}{c}\text { Thread density } \\
\text { Warp/Weft }(\mathrm{TPI})\end{array}$ & $\begin{array}{c}\text { Mass } \\
\left(\mathrm{g} / \mathrm{m}^{2}\right)\end{array}$ \\
\hline \hline GF108 & Glass fiber & 24.1 & 20.5 & $587.2 \pm 32.3$ & $60 / 47$ & 48 \\
GF1078 & Glass fiber & 19.8 & 13.1 & $392.0 \pm 34.4$ & $54 / 54$ & 48 \\
GF1080 & Glass fiber & 21.5 & 18.9 & $545.6 \pm 24.5$ & $60 / 47$ & 48 \\
GF1280 & Glass fiber & 13.1 & 14.1 & $304.0 \pm 17.2$ & $60 / 60$ & 56 \\
\hline K120-CST & Plain Kevlar & 4.2 & 3.9 & $79.7 \pm 3.6$ & $34 / 34$ & 60 \\
K120-EAS & Plain Kevlar & 2.2 & 1.8 & $57.0 \pm 2.0$ & $34 / 34$ & 60 \\
K120-13 & Coated Kevlar & N/A & 0.53 & $21.8 \pm 5.9$ & $34 / 34$ & 68 \\
K120-14 & Coated Kevlar & N/A & 0.95 & $15.1 \pm 5.6$ & $34 / 34$ & 74
\end{tabular}

Table 1. The properties of the tested porous fabrics. 


\section{B. Aeroacoustic testing}

Previous investigations have shown that the WJT is a good candidate to investigate scrubbing, and surface roughness noise sources $19[20$ In this tunnel, a 1/2-inch tall nozzle blows air over a flat plate, hence its name wall jet, which results in a self-similar flow over the plate 2425 The boundary layer has two main flow regions, the near-wall flow exhibits the properties of a canonical zero pressure gradient turbulent boundary layer until a maximum velocity is reached in the boundary layer profile. Above this point, a two-dimensional shear layer flow dominates, which is characterized by energetic, large-scale structures.

The rig used to assess the acoustic properties of the Kevlar fabrics is depicted in Fig. 1. The fabrics were mounted on a fabric tensioner, made by Eino Diamondchase, and were brought into a tension of $1500 \pm 200 \mathrm{~N} / \mathrm{m}$ measured using a Newman ST1E tension meter. The tensioning frame was flush-mounted to a 2 -ft. $\times 2$-ft. aluminum plate. The area of the fabric exposed to the flow was 15 -in. $\times 15$-in, see Fig. 2 . A B\&K type 4938 1/4-in. microphone was positioned below the center of the tensioned fabrics with a separation distance of 8-in. Given the high-frequency nature of the fabrics self-noise tested, the microphone was readily in the acoustic far field. The microphone was calibrated using a pistonphone calibrator on a daily basis. The flow speed of the jet in the wind tunnel was set to the maximum available, i.e. $u_{j e t}=70 \mathrm{~m} / \mathrm{s}$, during the self-noise measurements. This resulted in a flow passing over the tested fabrics with a maximum speed of approximately $25 \mathrm{~m} / \mathrm{s}$, corresponding to a Mach number of $M \approx 0.08$.

To obtain transmission loss data, a small, acoustically lined box was built of medium-density fiber (MDF) boards with overall dimensions of 19 -in. $\times 19$-in. $\times 24$-in. The interior of the box was lined with an acoustically absorbent 1-in. thick melamine foam. The estimated cut-off frequency of the acoustic box is $1-2 \mathrm{kHz}$. During the transmission loss measurements, the box was placed inside the anechoic chamber of the wall jet wind tunnel with acoustically absorbing foams placed over the wall jet plate. During the transmission loss tests, a Visaton FRS-8 speaker was mounted above the box and was fed with a white-noise signal. The B\&K type 4938 1/4-in. microphone was mounted inside the small acoustically lined box. Two measurements were performed. First, the opening was left open, and second, the opening was covered with a fabric sample. To quantify the efficacy of the instrumentation in determining the transmission loss, the acoustic loss of a Mylar sheet was also measured. For this configuration, a closed-form solution is available for the transmission loss, which is given in Section III.

In addition to the previously listed experiments, the surface pressure fluctuations were also measured. Two configurations were tested. First, the microphone was located upstream of the tensioning frame and then downstream of the K120-CST fabric. Second, the test rig was replaced by a plain aluminum plate and the microphone was placed at the same downstream position as before. An 1/8-inch, type 4138 B\&K microphone was flush mounted to the surface in each case. This transducer was used under a pinhole configuration to quantify the surface pressure spectrum and a dynamic calibration of the microphone was carried out a-priori from which the response of the transducer was determined. The Helmholtz effect of the cavity below the pinhole was compensated during post-processing ${ }^{26}$ of the data. Data was collected using a pair of B\&K LAN-XI cards for a time span of 32 seconds at a sampling rate of $2^{17}$ Samples/s (i.e. 131,072 Samples/s). During the post-processing of the data, the frequency resolution was set to $64 \mathrm{~Hz}$ using $50 \%$ of block overlapping and Hanning windowing during the Fourier transform calculations.

\section{Optical measurements}

To quantify the open area ratio of the fabrics experimentally, a YSC Technologies HD807 digital microscope was used to capture images of the fabrics. After placing a dark (black) background behind the fabrics, the pores (i.e. open area) were observed in the microscopic images as pixels with low intensity, while the majority of the yarns were observed in the images as high-intensity pixels. Through digital image processing, it was possible to identify and count the number of pixels representing the open areas, i.e. pores. The ratio between the number of these identified (masked) pixels and the overall number of pixels then defined the measured OAR, which is listed in Table. 1. A sample microscopic image of the K120-EAS fabric with the pores masked red is shown in Fig. 3 while the microscopic images of the glass fiber fabrics are shown in Fig. 4 and the Kevlar fabrics are shown in Fig. 5. 


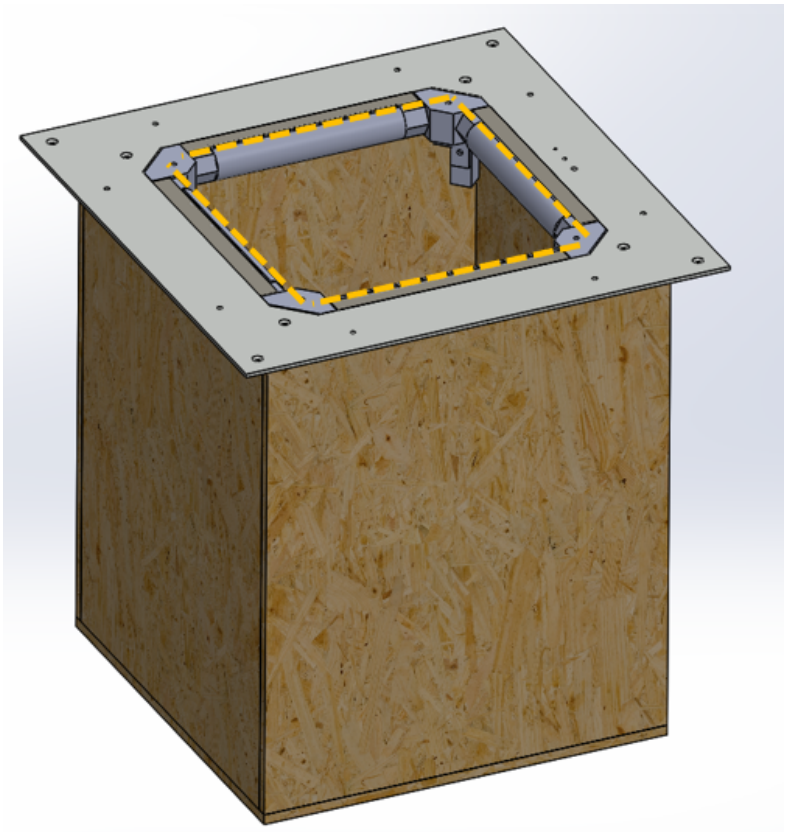

Figure 1. The CAD representation of the rig used to assess the aeroacoustic properties of porous fabrics. The dashed lines indicate the area occupied by the fabrics.

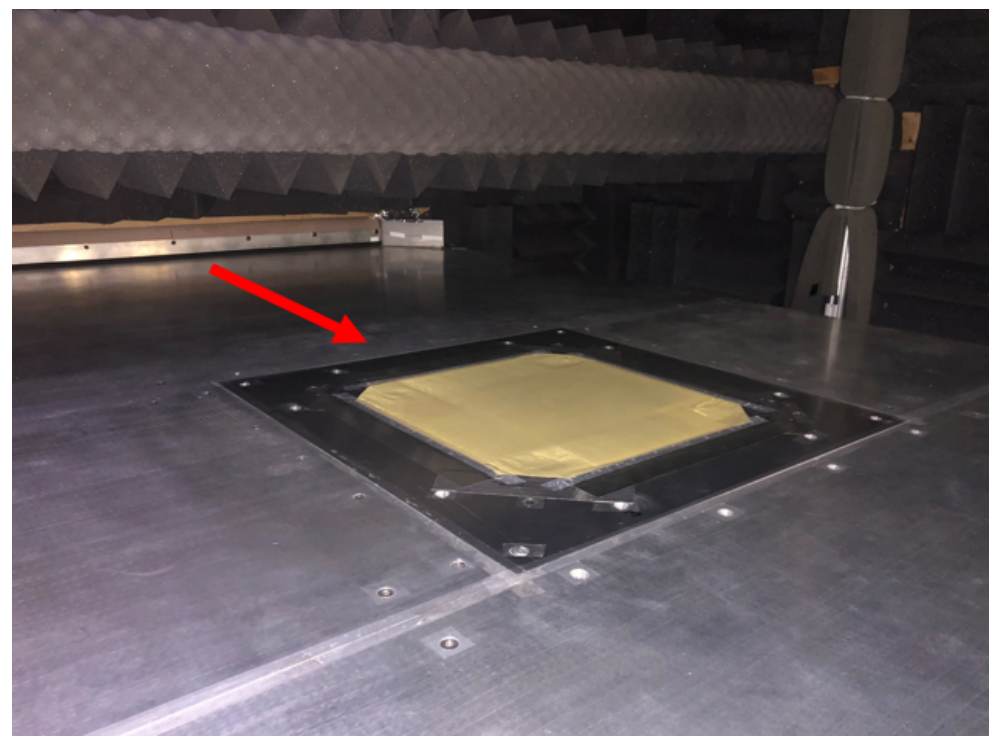

Figure 2. The rig mounted to the wall jet plate in the wall jet wind tunnel. The red arrow indicates the flow direction over the fabric. 

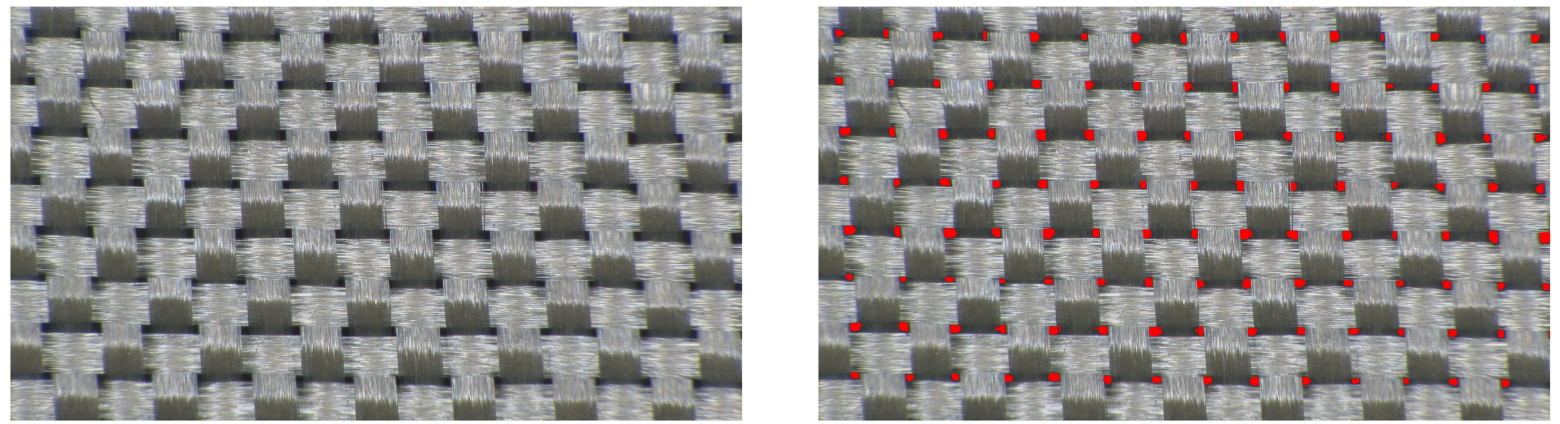

Figure 3. Sample microscopic image of K120-EAS fabric (left) with the pores marked red (right).

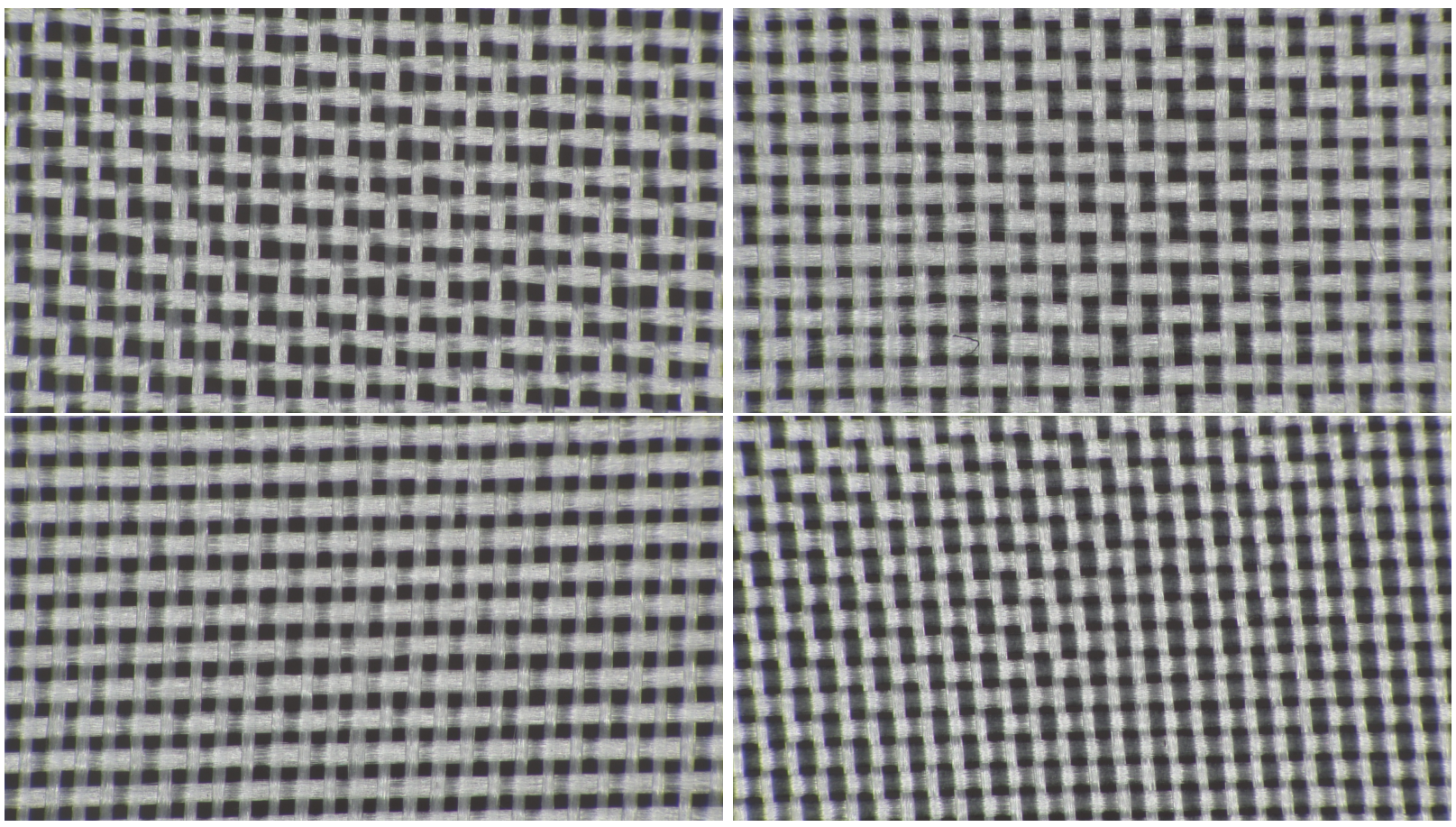

Figure 4. Microscopic images of glass fiber fabrics: G108 (top left), G1078 (top right), G1080 (bottom left), and G1280 (bottom right). 


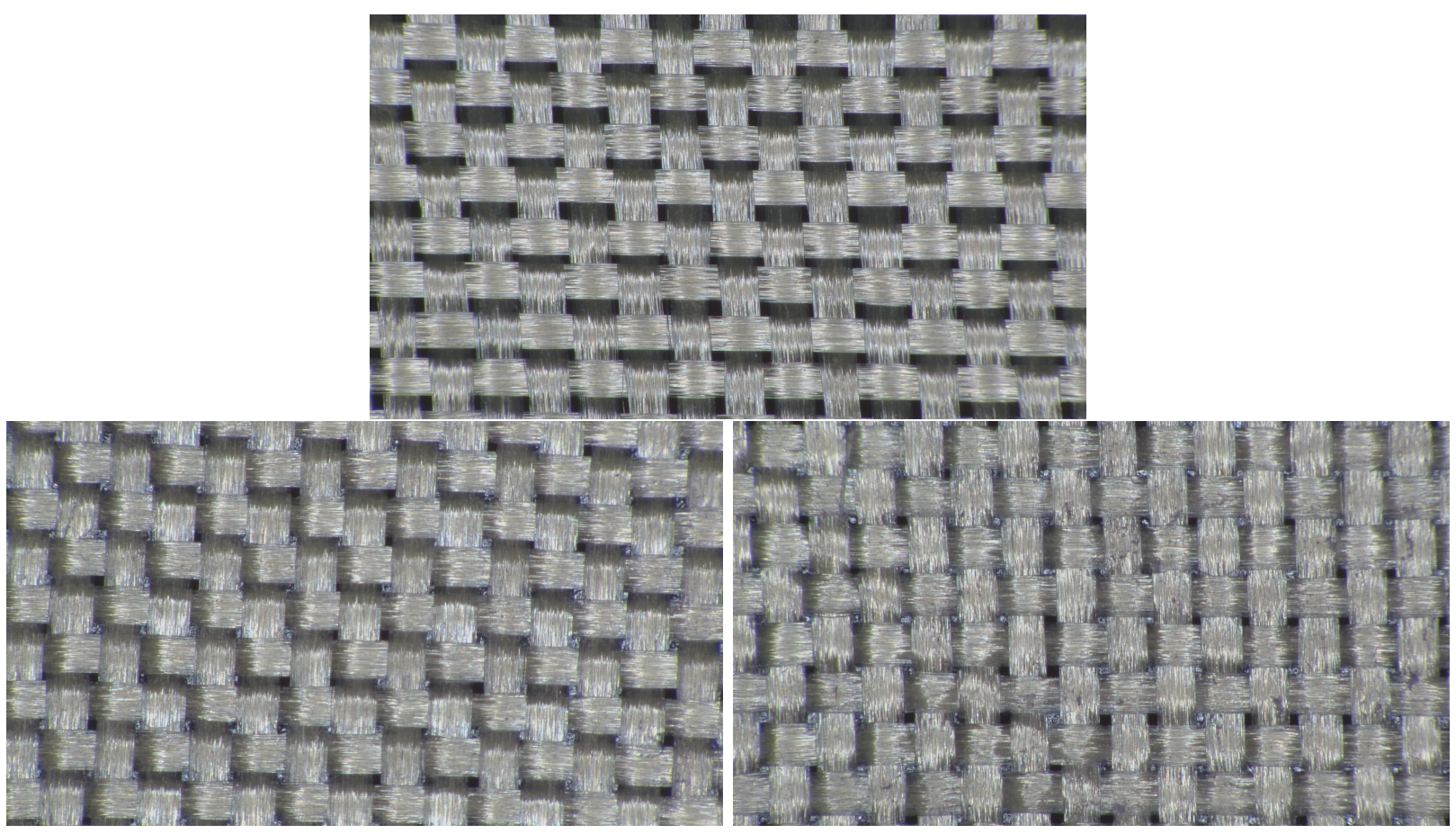

Figure 5. Microscopic images of Kevlar fabrics: K120-CST (top), K120-13 (bottom left), and K120-14 (bottom right).

\section{Results}

This section presents the results obtained from the three types of experiments. First, the transmission loss results are presented, followed by the self-noise generated by the different fabrics. Finally, the surface pressure measurements are shown to assess the effect of the fabrics on the boundary layer passing over them.

\section{A. Transmission loss}

Acoustic loss measurements may be considered a common type of acoustic testing. Such tests are most often performed for materials with high insertion loss. There, the experimental signal-to-noise ratio, i.e. drop in acoustic levels once the material is introduced, usually exceeds $10 \mathrm{~dB}$. Considering previous results of Kevlar transmission loss measurements, $12 \sqrt[213]{13}$ it was found that the acoustic loss of Kevlar does not exceed $5 \mathrm{~dB}$ at $20 \mathrm{kHz}$. This makes the quantification of TL rather challenging experimentally, especially at lower frequencies (below $1 \mathrm{kHz}$ ).

Acoustic models are readily available in the literature ${ }^{27}$ which provide a closed form solution for estimating transmission loss of thin materials. These models are often derived for a thin sheet material (or interface) in response to one dimensional time harmonic acoustic waves with normal incidence. However, a limited number of models are available for the case where porous materials or fabrics are tested because the fundamentals of how the sound propagates through such materials is fundamentally different from the case when the sound waves pass through a solid object. In the latter case, the loss is controlled by the mechanical interaction between the sound waves and the material. In case of porous fabrics, however, the mechanical interaction between the porous fabric and the sound waves is significantly reduced due to the presence of the pores. Namely, the sound waves can pass through the pores of the material significantly easier. This makes the direct comparison of currently available TL models with the TL results of porous fabrics presented here less relevant.

The transmission loss $(T)$ was calculated from the experimental data as

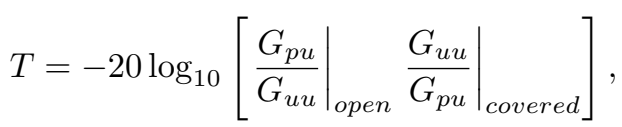


where $G_{a b}$ refers to the cross spectrum calculated between signals $a$ and $b$, the microphone pressure signal is denoted as $p$, the white noise signal fed to the speaker is represented by $u$ and the subscript "open" refers to data obtained with the cavity of the test rig being open while "covered" refers to the cavity of the test rig covered with the tested materials.

First, the transmission loss of a thin Mylar sheet is investigated to assess the capabilities of the transmission loss test rig. A closed-form solution for TL is readily available from Blackstock ${ }^{27}$ which quantifies the transmission loss of a thin sheet material in response to one-dimensional time-harmonic acoustic waves with normal incidence. In the case of Mylar, the transmission loss is mass-controlled and the expression simplifies to

$$
T=-20 \log _{10} \frac{2}{2+i \omega l \frac{\rho_{m}}{\rho_{0} c_{0}}},
$$

where $i$ is the imaginary unit, $\omega=2 \pi f, f$ is frequency, $\rho_{m}=1.38 \mathrm{~g} / \mathrm{m}^{3}$ is the density of the Mylar, $l=0.25 \mathrm{~mm}$ is the thickness of the Mylar, and $\rho_{0}$ and $c_{0}$ are the density of air and speed of sound in air, respectively.

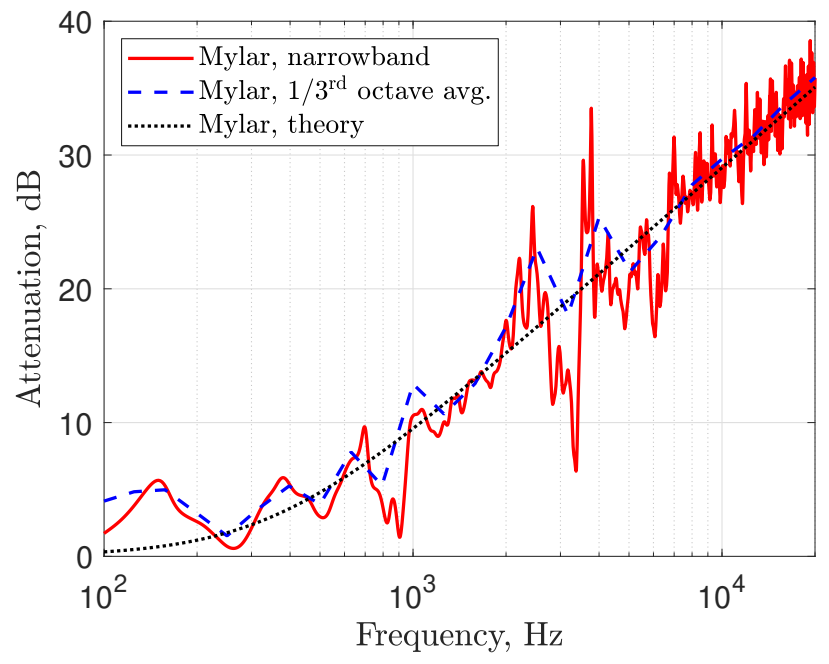

Figure 6. Experimental and theoretical transmission loss results obtained for a thin Mylar sheet.

The transmission loss results obtained for the Mylar sheet are shown in Fig. 6 alongside with the TL predicted using Eq. 22. The TL averaged over $1 / 3^{\text {rd }}$ octave bands are also shown in the figure to improve the accuracy of the TL results. The following observations can be made. First, the trend of the TL data agrees well with the theoretical prediction, which clearly indicates an increase of TL with frequency, particularly at high frequencies. This increasing trend of TL is due to the mass-controlled nature of the interaction between the sound waves and Mylar. The narrowband TL data shows larger reflections (uncertainty) at lower frequencies $(f<10 \mathrm{kHz})$, but above this threshold, the spread of the results decreases. There are a few distinct peaks in TL in the range of $3-4 \mathrm{kHz}$. Considering the estimated cut-off frequency of the small acoustically lined box $(1-2 \mathrm{kHz})$ and the change the introduction of the Mylar sheet causes to the acoustic boundary conditions of the box suggest that the TL data below $4 \mathrm{kHz}$ increases, particularly when the reflected sound waves from the material tested is large. Considering the lower mechanical interaction present between the porous fabrics and sound waves compared to the interaction of sound and Mylar, it is assumed that the internal reflection-related uncertainty is lower when pores are present. In other words, the internally reflected sound levels are anticipated to be lower for porous fabrics than for Mylar. Finally, a good agreement is found between the $1 / 3^{\text {rd }}$ octave averaged data and the theoretical TL values. For these reasons, only the $1 / 3^{\text {rd }}$ octave averaged TL results of the porous fabrics are shown in the following discussions.

The transmission loss results obtained for the glass fiber fabrics are shown in Fig. 7. As can be observed, the TL of these fabrics are extremely low, namely, the results remain less than $0.5 \mathrm{~dB}$ below $20 \mathrm{kHz}$. The uncertainty of the TL experiments was found to be approximately $\pm 0.1 \mathrm{~dB}$ from repeatedly measuring the acoustic signature of the configuration where no fabric was present over the cavity of the test rig. Based on this, the signal-to-noise ratio improves at high frequencies, particularly above $10 \mathrm{kHz}$. Here, fabrics show an increasing TL in the order of GF1080, GF108, GF1280, and GF1078. Considering their measured OAR 


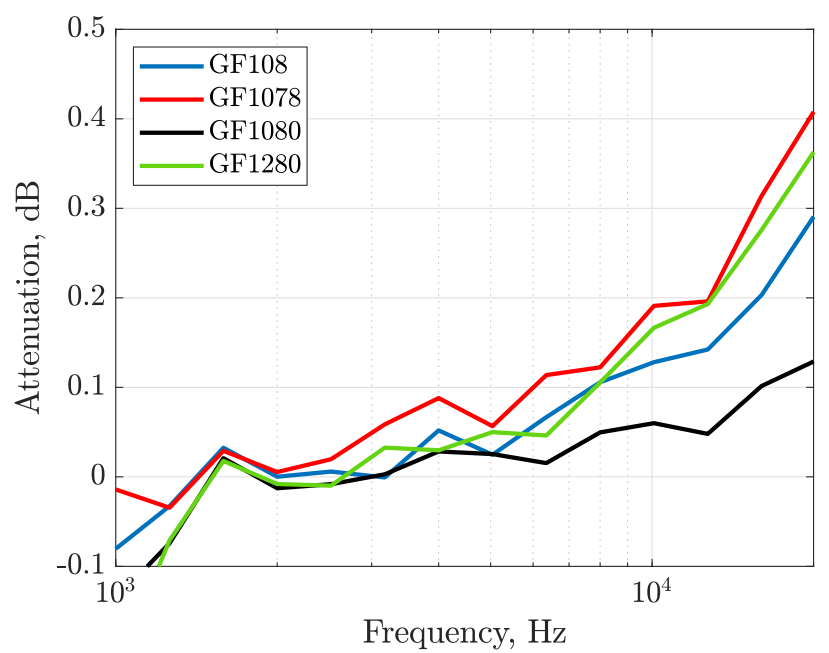

Figure 7. One-third octave band averaged transmission loss results obtained for the glass fiber fabrics.

values, this corresponds to OARs of $18.9 \%, 20.5 \%, 14.1 \%, 13.1 \%$. Even despite the relatively poor signalto-noise ratio (due to the extremely low TL), the trend suggests that a lower OAR will result in a higher transmission loss.

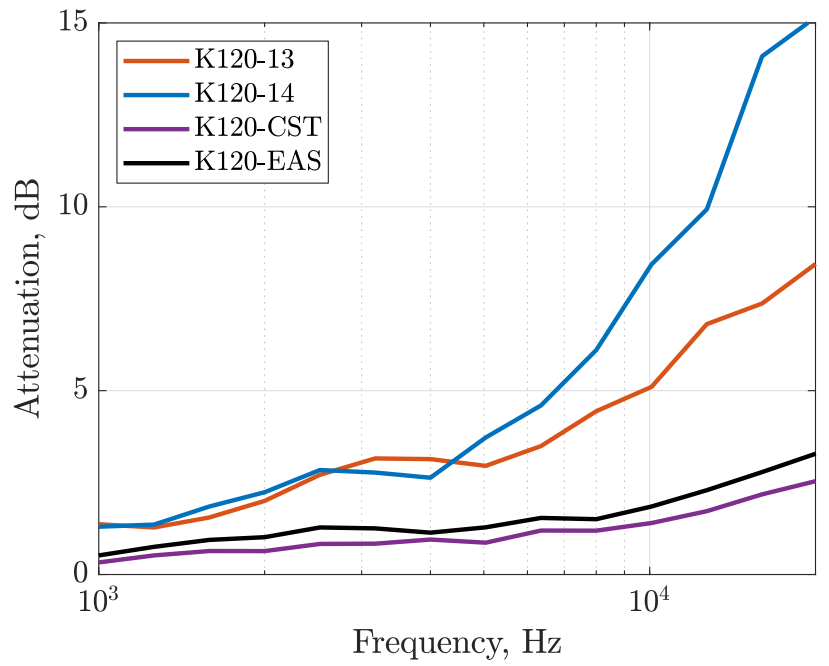

Figure 8. One-third octave band averaged transmission loss results obtained for the Kevlar fabrics.

Similar observations can be made by observing Fig. 8 where transmission loss results of the plain and coated Kevlar fabrics are shown. The plain Kevlar fabrics (K120-CST and K120-EAS) have an identical mass per unit area $(m)$ and thread density (TPI) and they only differ in OAR. Kevlar with a lower OAR shows a higher TL, which is also in agreement with our previous findings on the TL of glass fiber fabrics. From this, we may conclude that the OAR is the major factor in determining TL, particularly at high frequencies. When considering the coated Kevlar fabrics (K120-13 and K120-14), there is a well visible increase at high-frequencies (above $10 \mathrm{kHz}$ ), which is in agreement with our expectations, given their lower OAR. Additionally, there is a broadband increase in TL below $10 \mathrm{kHz}$. This may be explained by the increase in mass per unit area. The two coated fabrics, K120-13 and K120-14 have a higher $m$ by $13.3 \%$ and $23.3 \%$ compared to K120-EAS, respectively. As these fabrics have very small OAR $(<1 \%)$, their behavior may be compared to the model previously presented for the case of the Mylar sheet. Observing Eq. 2, it can be seen that a higher mass per unit area (i.e. sheet density) results in a TL curve where the mass-control nature of the interaction becomes significant at a lower frequency. In fact, a similar observation can be made for 
K120-13 and K120-14 where the TL begins to increase from $4 \mathrm{kHz}$ and $6 \mathrm{kHz}$, respectively.

Based on these observations, we can conclude that the acoustic loss of the commercially available Kevlar fabrics is dictated primarily by their OAR and secondarily by their mass per unit area. It was also seen that a very low OAR (see K120-13 and K120-14, in particular) shows a significant increase in TL. Therefore, when considering an acoustically ideal fabric, the OAR shall be maintained around a few percent to sustain acceptable, say less than 5-6 dB, TL at high frequencies.

\section{B. Self-noise}

Results of fabric self-noise are shown in Fig. 9 for all fabrics listed in Table1. The results can be categorized into three sets, namely, all glass fiber fabrics, both plain Kevlar fabrics and both coated Kevlar fabrics observe similar acoustic responses. This observation is also labeled in Fig. 9. All results shown were acquired at the maximum available flow speed of the wall jet wind tunnel, while the microphone was placed at an identical separation distance from the fabrics in the quiescent air. While the maximum jet speed in the tunnel is $70 \mathrm{~m} / \mathrm{s}$, this corresponds to a Mach number over the fabric samples of about $M \approx 0.08$. The maximum jet speed was necessary to obtain a good signal-to-noise ratio (self-noise to background noise) and to keep the aeroacoustic excitation of the fabrics identical, as the hydrodynamic pressure field passing over the fabrics is responsible for the pumping of air through the pores in the fabric.

For all cases where the fabric density differs in the warp and weft directions, the higher thread count was positioned perpendicular to the mean flow. Figure 9 also presents the background noise of the facility which was obtained by replacing the test rig with a flat plate (aluminum plate) and maintaining the location of the microphone. The background noise results reveal that the electrical noise of the data acquisition chain increases toward the Nyquist frequency $(65.5 \mathrm{kHz})$ and becomes comparable to the fabrics self-noise at approximately $50 \mathrm{kHz}$.

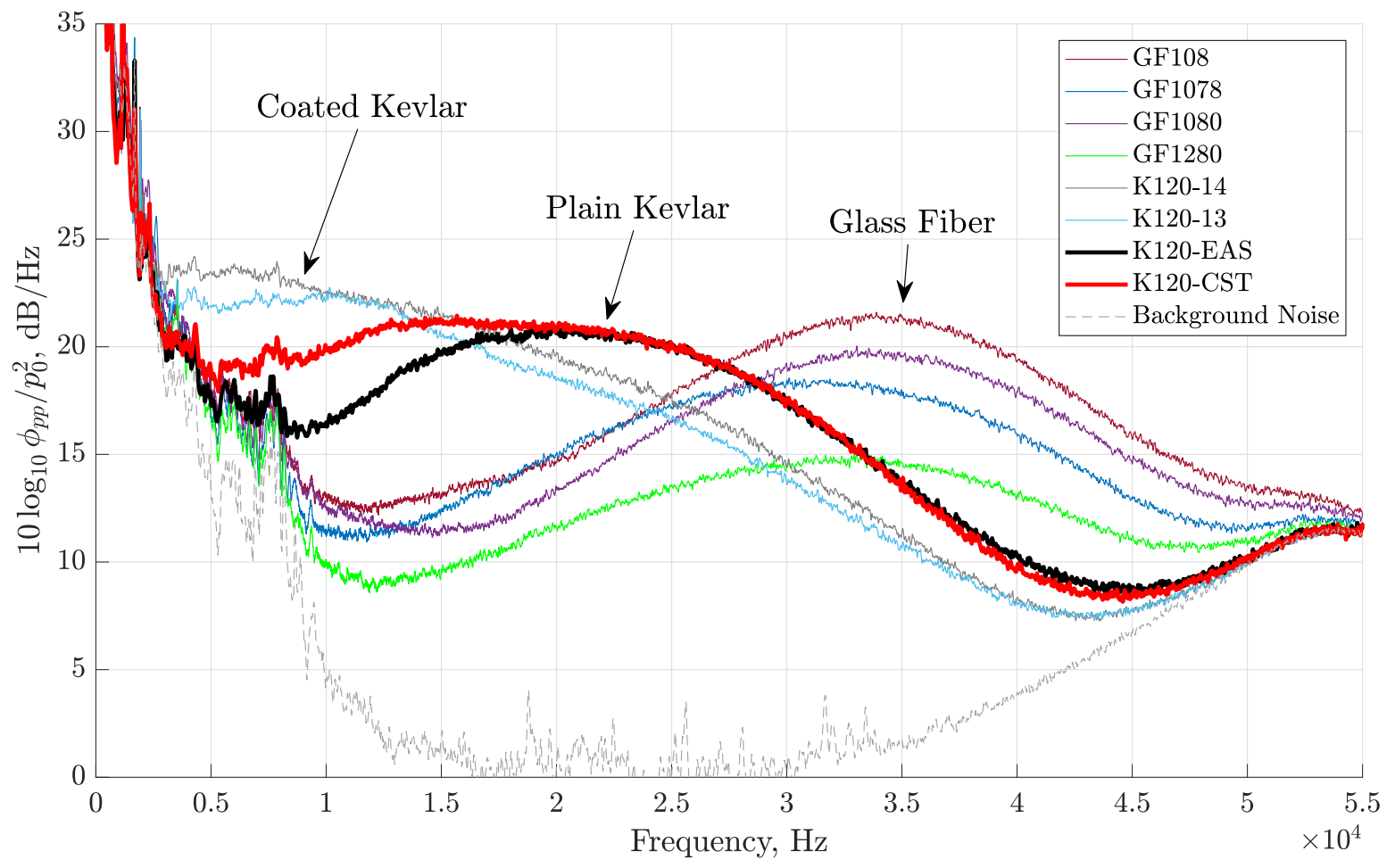

Figure 9. Narrowband self-noise data obtained for the porous fabrics.

Bahr et al ${ }^{[12}$ presented the acoustic signature of a Kevlar fabric that is identical to K120-CST at a similar Mach number. A good agreement is found between the acoustic signature of the plain Kevlar fabrics presented by Bahr et al. 12 and K120-CST in Fig. 9. Namely, the frequency responses of the fabrics are broadband in nature and span between approximately $7 \mathrm{kHz}$ and $40 \mathrm{kHz}$ in Fig. 9, while it spans between approximately $7 \mathrm{kHz}$ and $30 \mathrm{kHz}$ in Ref. ${ }^{12}$ When comparing K120-EAS to K120-CST, a near-identical 
acoustic response is seen above $20 \mathrm{kHz}$, but K120-CST produces more self-noise at lower frequencies (below $15 \mathrm{kHz}$ ) than K120-EAS. It may be concluded that the larger porosity of K120-CST is responsible for this increase.

The self-noise of the glass fiber fabrics is now considered, see Fig. 9. These fabrics differ from Kevlar in two main aspects, first is their significantly higher OAR (14-20\%) and increased (about double) thread density (54-60 TPI). The frequency span of the glass fiber fabrics self-noise is also broadband in nature and it is found over higher frequencies than that of Kevlar. The self-noise of the glass fiber fabrics span between $15 \mathrm{kHz}$ and $50 \mathrm{kHz}$ and they peak approximately at the same frequency, about $35 \mathrm{kHz}$. The only exception from this observation is GF1078, whose self-noise peaks at about $30 \mathrm{kHz}$. Another important observation is that their maximum self-noise ranges from 15-20 dB. Unfortunately, their OAR is high enough for air to travel across the materials at a significantly lower pressure loss, therefore, the wall jet flow could easily pass through these fabrics during testing. Hence, the exciting hydrodynamic pressure field above the fabrics was not identical to that of the Kevlar fabrics. This explains the wide range of self-noise levels and also suggests that the OAR contributes to the acoustic level of self-noise. Considering their rather similar acoustic response in terms of frequency span, this suggests that the frequency at which the broadband self-noise is observed is dictated by a material property that is similar across these fabrics. Most probably, this is due to their rather similar thread density. This assumption may be supported by the fact that the hydrodynamic pressure field passes over a higher number of threads per unit length whilst the convection velocity of the flow remains relatively constant. Another supporting evidence to this observation is the slightly different acoustic response of GF1078, which has a lower thread density (54 TPI) than the rest of the glass fiber fabrics. With this assumption, we may conclude that a higher thread density will shift the self-noise response of the porous fabrics to higher frequencies.

Finally, we investigate the acoustic response of the coated Kevlar fabrics, see Fig. 9. Applying spray-on glue to the K120-EAS fabric resulted in a random blockage of pores (see Fig. 5), which means that the interaction between the hydrodynamic pressure field passing over the fabric and the pores became nonperiodic or in other words random (broadband) in the spatial (wavenumber) domain. This may also mean that their thread density, which previously determined the number of pores per unit length as well, is not identical to that of K120-EAS from a pumping-excitation perspective. Also, their mass per unit area was increased by the addition of glue. Two observations can be made regarding their self-noise. First, an increase of self-noise below $15 \mathrm{kHz}$ is observed, and second, an approximately constant shift to lower frequencies can be seen when comparing to the plain Kevlar fabrics. From this, we may assume that their self-noise became more broadband in nature which could not be captured below $5 \mathrm{kHz}$ due to the comparable level of background noise. This observation may be confirmed in a future experiment by placing the current rig into the Stability Wind Tunnel where higher flow speeds can be reached, in turn increasing the self-noise levels, as was shown by Bahr et al ${ }^{[12}$ These observations suggest that the periodic pore distribution is responsible for the frequency span of porous fabrics. However, nearly eliminating the pores $(<1 \%)$ still results in a scrubbing-like self-noise again confirming their responsibility in the self-noise generation of porous fabrics. Finally, increasing the mass per unit area does not seem to have a significant, well distinguishable effect on the aeroacoustic response of porous fabrics.

From the results presented thus far, a few conclusions may be made by considering the joint effect of transmission loss and self-noise. Namely, when combining these properties as a penalty in signal-to-noise ratio in aeroacoustic experiments, we may identify two potential ways of improving their applicability. First, introducing a random distribution of pores can reduce the self-noise at high frequencies and shift it to lower frequencies but this was found to significantly increase the transmission loss $(15 \mathrm{~dB})$ at high frequencies $(20 \mathrm{kHz})$, see Fig. 8. Second, increasing the thread density while maintaining the OAR may be expected to shift the self-noise to higher frequencies and sustain an acceptable level of transmission loss.

\section{Surface pressure fluctuations}

As was seen in previous studies and through the self-noise results presented thus far, the hydrodynamic pressure field passing over the fabrics can be seen as the excitation function to the self-noise of the porous fabrics. Therefore, quantifying the surface pressure fluctuations (as a footprint of the hydrodynamic pressure field) may shed light on the interaction between the fabrics and the hydrodynamic pressure field within the boundary layer. For this reason, the power spectral density of the surface pressure fluctuations $\left(\phi_{p p}\right)$ was obtained upstream and downstream of the K120-CST fabric and was also measured at an identical downstream position using a hard wall (flat plate) configuration. This enables us to assess the effect of 
Kevlar on $\phi_{p p}$ and compare this effect to the undisturbed case.

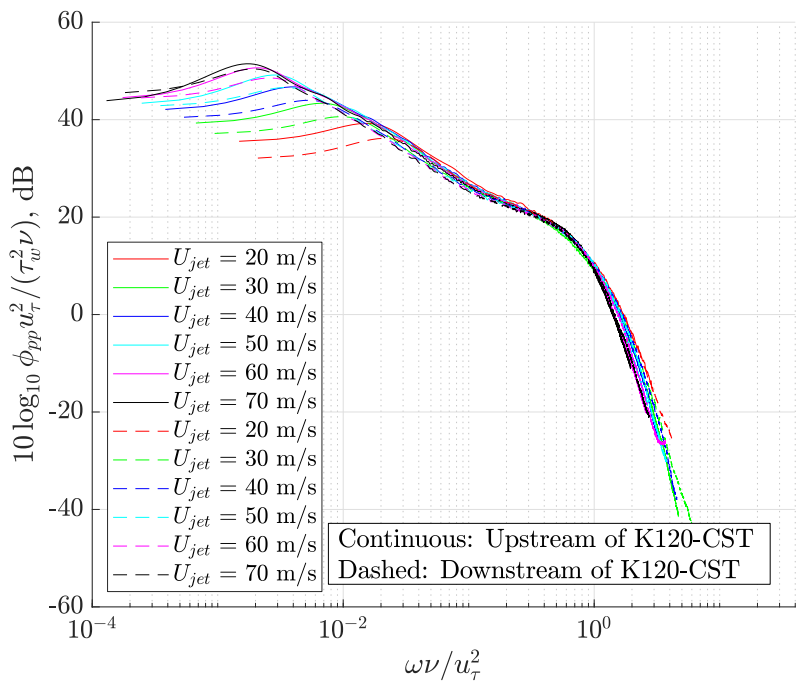

(a)

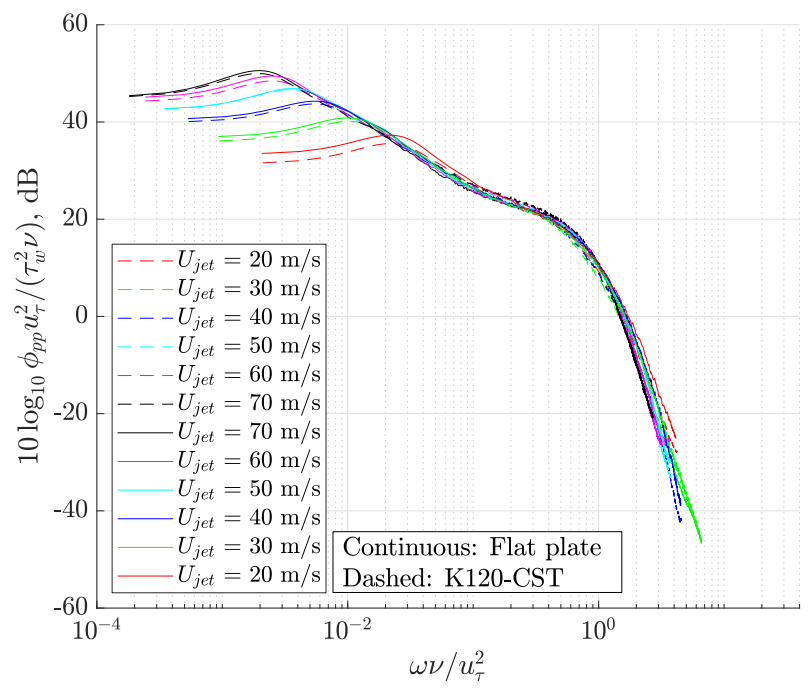

(b)

Figure 10. Surface pressure power spectral density measured upstream and downstream of the rig test area.

Figure 10 presents the normalized $\phi_{p p}$ results obtained for various nozzle jet velocities $\left(U_{j e t}\right)$ with the K120-CST mounted in the test rig (Fig. 10(a)), and the test rig replaced by a flat plate (Fig. 10(a)). The friction velocity $\left(u_{\tau}\right)$ and wall shear stress $\left(\tau_{w}\right)$ were determined for the center of the fabric area from using the facility calibration data presented in Ref., 21 while air viscosity $(\nu)$ and density $\left(\varrho_{0}\right)$ were determined from ambient air conditions recorded simultaneously to the acoustic measurements. Scaling the surface pressure spectrum with inner boundary layer time scales $\left(\nu / u_{\tau}^{2}\right)$ and pressure scales $\left(u_{\tau}^{2} /\left(\tau_{w}^{2} \nu\right)\right)$ provides a good collapse of the data in the mid and high-frequencies. As may be expected, the low-frequency portion of the spectrum changes with streamwise location and with flow speed.

Figure 10(a) compares the results obtained upstream and downstream of the Kevlar fabric. An important observation from the results is that the Kevlar fabric does not have any quantifiable effect on the results. In other words, the pores do not seem to alter the pressure spectrum of the mean flow when no pressure difference is present between the two sides of the fabric. This observation strongly suggests that Kevlar is a good candidate for separating the flow from quiescent conditions without affecting the hydrodynamic pressure field.

Figure 10(b) compares results obtained downstream of the Kevlar mounted in the test rig and the test rig replaced by a flat plate while the microphone was positioned at an identical streamwise location. Similar observations can be made regarding Fig. 10(b) as before, namely, a good collapse is observed while no significant change is found between the two sets of data (without Kevlar, with Kevlar). Again, these results confirm that the interaction between the hydrodynamic pressure field and Kevlar is minimal when no pressure difference is present on the two sides of the fabric. When a pressure difference is introduced, as it would be present in a HAWT configuration, one may expect that the boundary layer will undergo similar changes to that of previously seen in uniform flow suction of injection studies $28 \mid 29$ Future experiments focusing on the flow field are still needed to quantify the effect of a bias flow across Kevlar on the turbulent boundary layer. 


\section{Conclusions}

A set of porous fabrics are tested to assess their aeroacoustic properties with the aim of finding a fabric that may serve as an ideal aeroacoustic interface separating flow and quiescent conditions, in a similar manner that Kevlar is used in hybrid-anechoic wind tunnels. Experiments were conducted at the wall jet wind tunnel of Virginia Tech to characterize the transmission loss and self-noise of eight different porous fabrics. In addition, the effects of Kevlar on surface pressure fluctuations have also been investigated experimentally. For the purposes of the aeroacoustic tests, two separate test rigs were built to assess transmission loss and self-noise.

The eight fabrics tested in this study consisted of three major types: (a) four pieces of glass fiber fabrics, (b) two plain Kevlar fabrics, and (c) two modified Kevlar fabrics with a spray-on adhesive applied to a plain Kevlar. The effects of the following properties of the fabrics have been considered in the investigation of transmission loss and self-noise: open area ratio (OAR), defined as the porous area of the fabric to its overall area, thread density in the warp and weft direction (thread per inch, TPI), and the mass per unit area. The OAR of each fabric was also determined using digital microscopic imaging and image processing.

The transmission loss measurements confirmed that the pores serve as a low-resistance gateway to the sound waves, enabling a low transmission loss. The pores were found to separate the acoustic loss behavior of porous fabrics from a thin solid sheet of material, e.g. Mylar. This suggests that a fabric superior to the commercially available Kevlar fabrics still needs to rely on porosity to sustain an acceptable level of transmission loss. The exact level of porosity required may be determined using a tailor-made transmission loss model of porous fabrics, which may enable the independent variation of OAR without affecting thread density or mass per unit area.

The self-noise measurements of commercially available Kevlar were found to be in good agreement with previous measurements. The results suggest that the thread density determines the frequency span of porous fabric self-noise, while the open area ratio may have an impact on the generated sound levels. The fabric mass per unit area did not seem to significantly impact either transmission loss or self-noise.

Finally, the interaction between a turbulent boundary layer and a commercially available Kevlar fabric has been assessed. It was found that Kevlar did not have any quantifiable effect on the surface pressure fluctuations as it was observed to behave identically to a no-slip flat plate wall. This property of Kevlar also supports its use as an ideal interface between flow and quiescent air.

Our future investigations will focus on studying the fabrics under a higher Mach number flow, assessing the self-noise of different weave patterns with an ultimate goal of finding a better alternative to the currently available Kevlar fabrics.

\section{Acknowledgments}

The authors would like to thank the National Science Foundation, in particular Dr. Ron Joslin, for their support of this research under grant CBET-2012443. We would like to thank Danny Fritsch for his support with the surface pressure measurements.

\section{References}

\footnotetext{
${ }^{1}$ Devenport, W. J., Burdisso, R. A., Borgoltz, A., Ravetta, P. A., Barone, M. F., Brown, K. A., and Morton, M. A., "The Kevlar-walled anechoic wind tunnel," Journal of Sound and Vibration, Vol. 332, No. 17, 2013, pp. 3971-3991.

${ }^{2}$ Mayer, Y. D., Zang, B., and Azarpeyvand, M., "Aeroacoustic investigation of an oscillating airfoil in the pre-and post-stall regime," Aerospace Science and Technology, 2020, pp. 105880.

${ }^{3}$ Devenport, W., Bak, C., Brown, K., Borgoltz, A., Osterlund, J., and Davidsson, P., "Design and operation of hybrid aeroacoustic wind tunnels," Design and Operation of Aeroacoustic Wind Tunnel Tests for Group and Air Transport. NATO STO-EN-AVT-287, 2017.

${ }^{4}$ Szőke, M. and Azarpeyvand, M., "Reduction of the hydrodynamic noise on a beamforming array," Berlin Beamforming Conference, 2016.

${ }^{5}$ Horne, W. C. and Burnside, N., "Measurements of the Effects of Array Pattern Size and Windscreen Material on the Performance of a Wall-mounted Phased Microphone Array in a Hard-wall Wind Tunnel Using Enhanced In-flow Reference Sources," 25th AIAA/CEAS Aeroacoustics Conference, 2019, p. 2411.

${ }^{6}$ Todter, C., Marchetto, C., Robin, O., Berry, A., and Bremner, P., "Effect of screens and pinhole size on measured fluctuating surface pressures using a micro-electro-mechanical microphone array," INTER-NOISE and NOISE-CON Congress and Conference Proceedings, Vol. 254, Institute of Noise Control Engineering, 2017, pp. 136-143.
} 
${ }^{7}$ Fleury, V., Coste, L., Davy, R., Mignosi, A., Cariou, C., and Prosper, J.-M., "Optimization of microphone array wall mountings in closed-section wind tunnels," AIAA journal, Vol. 50, No. 11, 2012, pp. 2325-2335.

${ }^{8}$ Horne, W. C., Burnside, N. J., Schery, S. D., Podboy, G. G., Bahr, C. J., Stead, D., and Humphreys, W. M., "Effects of resistive windscreens and foam inserts on the acoustic response of an in-flow phased microphone array," AIAA Scitech 2021 Forum, 2021, p. 0217.

${ }^{9}$ Damani, S., Totten, E., Davies, L., Alexander, W. N., Devenport, W. J., Pearce, B. P., Shelley, S. R., Starkey, T. A., Hibbins, A. P., and Sambles, J. R., "Excitation of airborne acoustic surface modes driven by a turbulent flow," AIAA Aviation 2020 Forum, 2020, p. 2587.

${ }^{10}$ Szőke, M., Devenport, W., Borgoltz, A., Roy, C., and Lowe, T., "Advanced Boundary Simulations of an Aeroacoustic and Aerodynamic Wind Tunnel," NATO AVT-338 Research Specialists' Meeting on Advanced Wind Tunnel Boundary Simulation II., 2021.

${ }^{11}$ Lv, J., Wang, N., Liao, D., Yu, Y., and Gao, X., "Study on Acoustically Transparent Test Section of Aeroacoustic Wind Tunnel," Journal of Applied Mathematics and Physics, Vol. 6, No. 1, 2018, pp. 1-10.

${ }^{12}$ Bahr, C. J., Hutcheson, F. V., and Stead, D. J., "Assessment of unsteady propagation characteristics and corrections in aeroacoustic wind tunnels using an acoustic pulse," 2018 AIAA/CEAS Aeroacoustics Conference, 2018, p. 3118.

${ }^{13}$ Pascioni, K., Colangelo, A., and Cattafesta, L., "Acoustic Corrections for a Kevlar Wall Wind Tunnel Using a PulsedLaser Point Source," 24th International Congress on Sound and Vibration.

${ }^{14} \mathrm{Li}$, L., Liu, P., Guo, H., Hou, Y., Geng, X., and Wang, J., "Aeroacoustic measurement of 30P30N high-lift configuration in the test section with Kevlar cloth and perforated plate," Aerospace Science and Technology, Vol. 70, 2017, pp. 590-599.

${ }^{15}$ Debrouwere, M., "An Assessment of Acoustically Transparent Wind Tunnel Walls: For improving aero-acoustic measurements," 2013.

${ }^{16}$ Mayer, Y. D., Jawahar, H. K., Szőke, M., Ali, S. A. S., and Azarpeyvand, M., "Design and performance of an aeroacoustic wind tunnel facility at the University of Bristol," Applied Acoustics, Vol. 155, 2019, pp. 358-370.

${ }^{17}$ Ito, T., Ura, H., Nakakita, K., Yokokawa, Y., Ng, W., Burdisso, R., Iwasaki, A., Fujita, T., Ando, N., Shimada, N., et al., "Aerodynamic/aeroacoustic testing in anechoic closed test sections of low-speed wind tunnels," 16th AIAA/CEAS Aeroacoustics Conference, 2010, p. 3750.

${ }^{18}$ Brown, K., Brown, J., Patil, M., and Devenport, W., "Inverse measurement of wall pressure field in flexible-wall wind tunnels using global wall deformation data," Experiments in Fluids, Vol. 59, No. 2, 2018, pp. 25.

${ }^{19}$ Alexander, W. N. and Devenport, W. J., "Noise from boundary layer flow over fabric covered perforate panels," 20th AIAA/CEAS Aeroacoustics Conference, 2014, p. 2908.

${ }^{20}$ Alexander, W. N. and Devenport, W. J., "Noise Produced by Fabric and Wire Mesh Covered Panels in Low-Speed Anechoic Wind Tunnels," 21st AIAA/CEAS Aeroacoustics Conference, 2015, p. 3261.

${ }^{21}$ Kleinfelter, A. W., Repasky, R., Hari, N., Letica, S., Vishwanathan, V., Organski, L., Schwaner, J., Alexander, W. N., and Devenport, W. J., "Development and Calibration of a new Anechoic Wall Jet Wind Tunnel," AIAA Scitech 2019 Forum, 2019, p. 1936.

${ }^{22} \mathrm{Hu}$, J., Structure and mechanics of woven fabrics, Elsevier, 2004.

${ }^{23}$ Mohammadi, M., Banks-Lee, P., and Ghadimi, P., "Air permeability of multilayer needle punched nonwoven fabrics: Theoretical method," Journal of industrial textiles, Vol. 32, No. 1, 2002, pp. 45-57.

${ }^{24}$ Gersten, K., "The asymptotic downstream flow of plane turbulent wall jets without external stream," Journal of Fluid Mechanics, Vol. 779, 2015, pp. 351.

${ }^{25}$ George, W. K., Abrahamsson, H., Eriksson, J., Karlsson, R. I., Löfdahl, L., and Wosnik, M., "A similarity theory for the turbulent plane wall jet without external stream," Journal of Fluid Mechanics, Vol. 425, 2000, pp. 367-411.

${ }^{26}$ Fritsch, D., Vishwanathan, V., Lowe, K. T., and Devenport, W. J., "The Effect of Grazing Flow on Pinhole Condenser Microphones," AIAA Scitech 2021 Forum, 2021, p. 0130.

${ }^{27}$ Blackstock, D. T., Fundamentals of physical acoustics, Acoustical Society of America, 2001.

${ }^{28}$ Szőke, M., Fiscaletti, D., and Azarpeyvand, M., "Influence of boundary layer flow suction on trailing edge noise generation," Journal of Sound and Vibration, Vol. 475, 2020, pp. 115276.

${ }^{29}$ Szőke, M., Fiscaletti, D., and Azarpeyvand, M., "Uniform flow injection into a turbulent boundary layer for trailing edge noise reduction," Physics of Fluids, Vol. 32, No. 8, 2020, pp. 085104. 